\title{
Regresse und Routinen. Repliken auf Brandt und Jung
}

\author{
David Löwenstein, loewenstein@em.uni-frankfurt.de \\ In: Zeitschrift für philosophische Forschung \\ letzte Entwurfsfassung vom 20. Oktober 2017
}

\section{Einleitung}

Meine Auffassung von Know-how als Kompetenz soll sich zwischen Intellektualismus und Anti-Intellektualismus positionieren und die Einsichten beider Seiten auszunutzen, um die Probleme der anderen zu lösen. Wie Stefan Brandt und Eva-Maria Jung klar und überzeugend darlegen, wirft dieser Versuch jedoch einige miteinander verbundene Schwierigkeiten auf.

Wenn er, wie Brandt argumentiert, einer Variante des notorischen Regressproblems zum Opfer fällt, dann sitzt er hier mit dem Intellektualismus in einem Boot. Der wesentlich praktische Charakter von Know-how wird dann nicht mehr verständlich. In meinem Versuch, dieses Problem zu lösen, berufe ich mich wesentlich auf eine Analyse des vom Anti-Intellektualismus betonten Phänomens, dass Kompetenzen teils auch in völlig automatischen Akten ausgeübt werden können. Wenn Jung recht hat, dann zeigen sich hier jedoch einige Inkonsistenzen, ein Symptom einer systematischen „Hin- und Hergerissenheit" zwischen Intellektualismus und Anti-Intellektualismus (XX).

Ich freue mich sehr, diese Probleme im Lichte solch klarer und konstruktiver Kritik weiter durchdenken zu dürfen. Doch lassen Sie sich auch lösen? 


\section{Regresse}

Brandt formuliert zwei Regresseinwände. Der erste setzt bei der These an, dass die Kompetenz, A zu tun, die Kompetenz beinhaltet, Akte der Aktivität A zu bewerten. Doch jede solche Bewertungskompetenz beinhaltet dann qua Kompetenz ebenfalls eine Meta-Bewertungskompetenz, und immer so weiter. Der Kern meines Lösungsvorschlags bestand in einer Selbstreflexivitätsthese (vgl. KHC §4.6). Kurz skizziert: Zu einer Bewertungskompetenz gehört zwar stets eine Meta-Bewertungskompetenz, doch diese sind miteinander identisch. Zu bewerten und Bewertungen zu bewerten sind Ausübungen desselben selbstreflexiven Vermögens.

Wenn ich Brandt richtig lese, ist er mit dieser Lösung im Grunde einverstanden, schlägt aber vor, die Selbstreflexivitätsthese leicht abzuwandeln und das wichtigste meiner drei Argumente für diese These zu verbessern (XX). Sein Einwand lautet: Eine Bewertung - ein Urteil erster Stufe der Form (B 1) - impliziert zwar stets eine Bewertung einer solchen Bewertung als korrekt - ein Urteil zweiter Stufe der Form (B1'). Doch diese Urteile unterscheiden sich in ihren Gehalten, da der Bezug auf Bewertungen und der Begriff der

Korrektheit erst auf der zweiten Stufe vorkommen (XX). Nach Brandt wird aus meinem Argument erst eine Ebene weiter ein Schuh. Eine Bewertung zweiter Stufe impliziert nicht nur eine Bewertung dritter Stufe, sondern verwendet auch dieselben Begriffe und bezieht sich auf dieselbe Art Entität. Der Regress endet also erst deswegen, weil jede Meta-Bewertungskompetenz mit ihrer Meta-Meta-Bewertungskompetenz identisch ist (XX).

Dem kann ich gerne folgen, denn auch so wird das Problem gelöst. Dennoch möchte auch meine ursprüngliche Lösung gegen Brandts Kritik stark machen (vgl. KHC 135-137). Eine erststufige Bewertung der Form „Akt x in den Umständen C ist ein A-en der Qualität Q" lässt sich paraphrasieren als „Hinsichtlich der Aktivität A bewertet, hat Akt x in den Umständen C die Qualität Q“. Das heißt: Im Begriff „ein A-en der Qualität Q“ steckt implizit bereits der Bezug auf eine Bewertung als korrekt. Etwas als A-en 
zu bewerten, also als A-en statt als ein beliebiges anderes B-en (etwa als Fernschuss statt als Konfliktlösungsversuch), erfordert Bezug auf spezifische Korrektheitsbedingungen (die des A-ens statt des B-ens). Wenn das stimmt, fällt eine Bewertungskompetenz nach den von Brandt zugestandenen Kriterien am Ende doch mit ihrer Meta-Bewertungskompetenz zusammen. Aber wenn nicht, erreichen wir das Ziel ja auch eine Ebene weiter.

Brandts zweiter Einwand betrifft die Frage, was es heißt, dass kompetent Handelnde von einem Verständnis der betreffenden Aktivität geleitet sind. Meine These lautete, dass es hier auf die Vollzüge der zugehörigen Bewertungskompetenz ankommt. Doch dann ist jede Bewertungskompetenz qua Kompetenz ebenfalls von den Vollzügen ihrer Meta-Bewertungskompetenz geleitet. Auch hier scheint ein infiniter Regress unvermeidlich (XX).

Brandt schlägt vor, sich darauf zu beschränken, „dass die Kompetenz A zu tun, zwar die Kompetenz, Ausübungen von A zutreffend zu beurteilen, voraussetzt, nicht aber tatsächliche Vollzüge dieser Kompetenz." (XX) Das ist plausibel, lässt völlig aber offen, was es heißt, dass eine Person ein Verständnis einer Aktivität nicht nur hat (das mag ja auch aufs Verdauen zutreffen), sondern davon in ihrem Tun geleitet ist. Genau dies meint Ryle, wenn er von ,intelligentem‘ Tun spricht (vgl. KHC § 1.5).

Mein Lösungsvorschlag besteht in der Idee der Kultivation von Automatismen. Darauf bezieht sich Brandt gar nicht explizit, sondern merkt einzig an, dass ich nicht fordere, dass die betreffenden Bewertungen gleichzeitig mit den erststufigen Vollzügen getroffen werden. Wie ich zu zeigen versuche (vgl. KHC $\S \S 3.4,4.4-4.5)$, können sie genauso vorher oder auch erst danach stattfinden. Und sie können genauso explizit und intentional wie unbewusst und automatisch sein. Kurz gesagt: Entscheidend ist allein, dass sie insgesamt ein Teil des Bemühens sind, die eigenen Vollzüge des A-ens so zu gestalten, dass sie den Normen des A-ens möglichst gut entsprechen.

Folgt nun ein infiniter Regress? Etwas ist von den Vollzügen einer Bewertungskompetenz geleitet, wenn solche Bewertungen einen Beitrag zur Kul- 
tivierung der aktivierten Automatismen geleistet haben, gerade leisten oder noch leisten werden. Das setzt keine vorgängige unendliche Bewertungskette voraus, sondern allein die wesentliche Offenheit für einen kontinuierlichen Prozess des nachträglichen Bewertens, Revidierens und Weiterlernens.

Natürlich, auch die Kultivierung von Routinen und Automatismen muss irgendwann beginnen. Kompetenzen müssen erlernt worden sein, an einem wie auch immer vage bestimmten Zeitpunkt. Doch hier liegt kein problematischer Regress vor, sondern schlicht das Rätsel, an welcher Stelle das Verhalten und die Dispositionen eines Organismus derart sind, dass vollwertiges Wissen, Kompetenzen und Begriffen vorliegen. Diese Frage kann ich hier nicht beantworten (vgl. KHC §6.7), sodass Brandts zweiter Einwand in dieser Hinsicht völlig berechtigt ist. Doch dasselbe Problem stellt sich für alle Positionen in der Debatte um Know-how. Hier liegt also kein spezifischer Einwand gegen meinen Vorschlag vor, sondern eine weitere philosophische Projektbeschreibung, auf die alle verpflichtet sind.

\section{Routinen}

Meine Antwort auf das Regressproblem beruft sich wesentlich auf meine Überlegungen zu Automatizität und Kompetenz. Doch genau dafür identifiziert Jung gleich zwei Schwierigkeiten.

Ich behaupte, dass Automatismen nur dort Kompetenzen darstellen können, wo sie im Lichte eines Verständnisses der betreffenden Aktivität kultiviert sind. Doch Jung hat ein Gegenbeispiel vor, den „unerwünschten Ohrwurm", also eine Kompetenz, ein Lied zu singen, die unerwünscht ganz automatisch erworben wurde (XX). Das ist in der Tat ein gutes Beispiel, aber auch ein Grenzfall. Zur Kultivierung von Automatismen gehört nicht nur die Lerngeschichte, sondern auch die wesentliche Offenheit zum Weiterlernen (vgl. KHC $\S \S 2.4,3.4,4.4)$. Wo man sich in einem automatischen, gedankenlosem Tun wiederfindet, aber wie hier aktiv gegensteuert, liegt also zwar eine 
Fähigkeit vor, aber eher keine Kompetenz - ,eher', weil der Kompetenzbegriff vage Grenzen hat und je nach Kontext unterschiedlich anspruchsvoll ist (vgl. KHC §1.7). Einer Person, die beim Singen des unerwünschten Ohrwurms beginnt, aktiv die Melodie zu halten, lässt sich durchaus genuines Know-how zuschreiben, auch wenn dessen Erwerb notorisch schwer datierbar ist.

Das andere von Jung benannte Problem beginnt mit meiner These, dass es im automatischen Tun Beispiele für Ausübungen von Know-how gibt, die keine intentionalen Handeln sind (vgl. KHC §3.1). Das Paradebeispiel des automatischen Lesens einer Aufschrift (KHC 78) zeigt das unabhängig davon, wie intentionale Handlungen positiv bestimmt werden. Gleichzeitig schlage ich aber vor, die Ausübung von Know-how durch verantwortliche Kontrolle zu erläutern (vgl. KHC §4.4). Doch der Begriff der Kontrolle ist hier erst dann anwendbar, wenn zugestanden wird, dass einzelne Akte (wie automatisches Lesen) nur in vermittelter Weise kontrolliert sind. Hier diagnostiziert Jung eine Inkonsistenz. Wenn völlig automatische Akte genau dort Ausübungen von Know-how sind, wo sie auf diese Weise vermittelt kontrolliert sind: Wieso sind sie dann nicht aus genau demselben Grund auch intentional, ebenso etwa wie automatische Sub-Prozesse, etwa Muskelkontraktionen, intentional sind als Teil einer übergeordneten Handlung? (XX)

Diese Probemdiagnose trifft ins Schwarze. Doch im Grunde ist der genaue Ort des Begriffs der intentionalen Handlung sekundär für meinen Versuch, Know-how als Kompetenz verständlich zu machen. Daher könnte ich meine Position im Prinzip gut so erweitern, dass auch dies Fälle intentionalen Handelns sind. Jung schreibt: „Das Lesen des Schildes könnte beispielsweise auf die übergeordnete Intention bezogen werden, geschriebene Informationen aus der Umwelt aufzunehmen und auszuwerten." (XX) Das kommt mir plausibel vor, doch die Details sind natürlich kompliziert: Die Intention, Geschriebenes aufzunehmen, hat ja plausiblerweise nicht die Form eines strikten Allsatzes, sondern gilt eher ceteris paribus. Aber wenn sie sich in der Tat auf den in Frage stehenden Fall bezieht, liegen dort inkohärente Intentionen vor, denn 
dass ich diese Aufschrift in Wahrheit nicht lesen will, stand ja fest. Jung hat recht: Hier ist weitere Arbeit vonnöten, vor allem zum Begriff der intentionalen Handlung. 